\title{
Characterization of Aldolase from Methanococcus jannaschii by Gas Chromatography
}

\author{
Jeong E. Nam Shin, Mi-Jung Kim, Ji-ah Choi and Keun Ho Chun* \\ Department of Chemistry, Soongsil University, Dongakgu Sangdo 5 dong, Seoul 156-743, Korea
}

Received 12 April 2007, Accepted 21 May 2007

The products of reactions catalyzed by Methanococcus. jannaschii (Mj) aldolase using various substrates were identified by gas chromatography (GC). Although $\mathrm{Mj}$ aldolase is considered a fuculose-1-phosphate aldolase based on homology searching after gene sequencing, it has not been proven to be a fuculose-1-phosphate aldolase based on its reaction products. $M j$ aldolase was found to catalyze reactions between glycoaldehyde or $\mathrm{D}$, L-glyceraldehyde and DHAP (dihydroxyacetone phosphate). Before performing GC the ketoses produced were converted into peracetylated alditol derivatives by sequential reactions, i.e., dephosphorylation, $\mathrm{NaBH}_{4}$ reduction, and acetylation. By comparing the GC data of final products with those of standard alditol samples, it was found that the enzymatic reactions with glycoaldehyde, D-glyceraldehyde, and D, Lglyceraldehyde produced D-ribulose-1-phosphate, Dpsicose-1-phosphate, and a mixture of D-psicose and Ltagatose-1-phosphate, respectively. These results provide direct evidence that $M j$ aldolase is a fuculose-1-phosphate aldolase.

Keywords: Fuculose-1-Phosphate aldolase, Gas Chromatography, Methanococcus jannaschii

\section{Introduction}

The enzymatic aldol reaction is one of the essential bio reactions, and is highly efficient and stereoselective (Stephen et al., 2006). For these reasons, aldolases have long been of interest to synthetic chemists (Fessner et al., 2000; Espelt et al., 2005). Many studies have been conducted on various aldolases in terms of their characterization, mechanism elucidation, substrate specificity, and organic synthesis applications

*To whom correspondence should be addressed.

Tel: 82-2-820-0437; Fax: 82-2-824-4383

E-mail: kchun@ssu.ac.kr
(Wong et al., 1995; Dreyer et al., 1996, Kroemer et al., 2003; Calveras et al., 2006).

Recently, various heat stable aldolases from hyperthermophilic micro organisms have been found and investigated in depth, because several of them show enhanced stability and performances at high temperature, even in organic solvents (Yoon et al., 2001; Jeyakanthan et al., 2005). However, in terms of their synthetic purposes, they still have relatively narrow substrate specificities.

The rapid cloning of an aldolase homologue from Methanococcus. jannaschii (Mj), a hyperthermophilic microorganism, by over-expression was described by Choi et al. (Choi et al., 1998). The purified enzyme showed aldolase activity and was found to be unusually resistant to heat, denaturants, and organic solvents. However, no full characterization of the enzyme has been performed, although its enzymic activities have been estimated by monitoring DHAP consumptions, but this was not supported by an analysis of reaction products. $M j$ aldolase is considered to be a type II fuculose-1-phosphate aldolase based on its homology known fuculose aldolases, but no direct evidence on product identification is available. Thus, in the present study, the authors analyzed reaction products by gas chromatography (GC). GC has advantages for the identification of carbohydrate derivatives as only trace amounts of sample are required. Moreover, since DHAP, the major aldolase substrate, is relatively expensive, small-scale experimentation is more attractive. To analyze a ketose derivative, as is produced during aldolase, ketoses are reduced to alditol derivatives, and alditol poly hydroxy groups are protected prior to GC to produce volatile species. In addition, standard samples of known alditols are necessary to identify unknowns by comparing retention times.

\section{Materials and Methods}

DHAP was purchased from Sigma-Aldrich, or synthesized (Frank et al., 2004). MJ aldolase was produced as previously described 
(Choi et al., 1998). All chemicals and solvents were purchased from Sigma-Aldrich or TCI America.

General procedures used for $M j$ aldolase reactions, $\mathrm{NaBH}_{4}$ reduction, and acetylation. To reaction mixture in buffer $(1 \mathrm{ml}$, $\mathrm{pH}$ 8) containing $50 \mathrm{mM}$ DHAP, $125 \mathrm{mM}$ aldehyde, $0.1 \mathrm{mM}$ $\mathrm{ZnCl}_{2}$, and $100 \mathrm{mM}$ Tris- $\mathrm{HCl}$, was added $80 \sim 120 \mu \mathrm{Mj}$ of aldolase solution $(20 \mathrm{mg} / \mathrm{L})$. The reaction mixture was then stirred for $1 \mathrm{~h}$ at $37^{\circ} \mathrm{C}$. After checking product formation and the disappearance of DHAP by TLC (Merk, Kiesegel $60 \mathrm{~F}_{254}$, solvent : $n$-butanol : acetic acid $:$ water $=5: 3: 2), \mathrm{Ba}(\mathrm{OAc})_{2}(11.49 \mathrm{mg})$ was added dropwise until a white precipitation formed. After adjusting the $\mathrm{pH}$ to 7.5 , acetone $(2.4 \mathrm{ml})$ was added, and the reaction mixture was stirred for $24 \mathrm{~h}$ at $4^{\circ} \mathrm{C}$. The white precipitate so formed was separated by centrifugation and washed with acetone and ether several times. The precipitate was then dissolved in $0.5 \mathrm{ml}$ water containing 27 $\mathrm{mg}$ of Dowex resin $\left(50 \times 8, \mathrm{H}^{+}\right.$, Sigma $)$with stirring. After filtering off resin, the $\mathrm{pH}$ of the filtrate was adjusted to 5 with $2 \mathrm{~N} \mathrm{HCl}$ solution, and a trace amount of acid phosphatase (Sigma) was added to detach the phosphate group from the product. Without any purification, the reaction mixture was then treated with $\mathrm{NaBH}_{4}$ (7.5 $\mathrm{mg})$. After $10 \mathrm{~h}$ of stirring, the reaction flask was placed in an ice bath, and methanol $(1 \mathrm{ml})$ was added. The solvent was removed by vacuum distillation, and the remaining syrup was dissolved in a mixture of acetic anhydride $(0.5 \mathrm{ml})$ and pyridine $(0.5 \mathrm{ml})$. The reaction mixture was then stirred for $16 \mathrm{~h}$ at $120^{\circ} \mathrm{C}$, cooled to $0^{\circ} \mathrm{C}$, and $5 \mathrm{ml}$ of water was slowly added. After stirring for $1 \mathrm{~h}$ at room temperature, the reaction mixture was extracted with $\mathrm{CH}_{2} \mathrm{Cl}_{2}$ several times. The organic layers were collected, dried, filtered, and evaporated to yield a syrup-like product. Final separation was performed by silica gel chromatography.

Procedure for preparing standard alditol peracetate samples. Adonitol, D-arabitol, xylitol, allitol, D-talitol, D-sorbitol, D-mannitol, and dulcitol were purchased from Sigma-Aldrich or TCI America. Each of these $(0.5 \mathrm{~g})$ was acetylated using acetic anhydride $(25 \mathrm{ml})$ and pyridine $(25 \mathrm{ml})$ using the procedure mentioned. Final separations were performed by silica gel chromatography, and product structures were confirmed by NMR.

General procedure for GC. The GC unit used was a HP 5890 series II equipped with an FID detector and a DB23 capillary column. Helium was passed at $18 \mathrm{ml} / \mathrm{min}$, and the oven was set at $220^{\circ} \mathrm{C}$. To prepare sample solutions, $1 \mathrm{mg}$ of each sample was dissolved in $1 \mathrm{ml}$ of $\mathrm{CH}_{2} \mathrm{Cl}_{2}$, and $2 \mu \mathrm{l}$ was then injected.

\section{Results and Discussion}

It was considered that if $M j$ aldolase is a fuculose-1-phosphate aldolase, then it would convert DHAP and glycoaldehyde to D-ribulose-1-phosphate, and convert DHAP and D-glyceraldehyde into D-psicose-1-phosphate. Moreover, its action on D, L-glyceraldehyde should result in a mixture of D-psicose1-phosphate and L-tagatose-1-phosphate.

For GC analysis, these ketoses were dephosphorylated and converted into their corresponding alditol derivatives by $\mathrm{NaBH}_{4}$

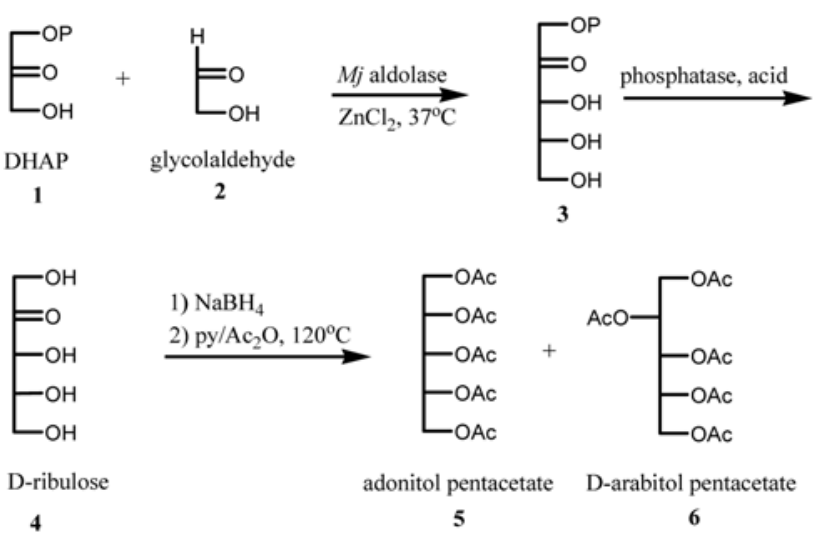

Scheme 1. $M j$ aldolase reaction with DHAP and glycoaldehyde.

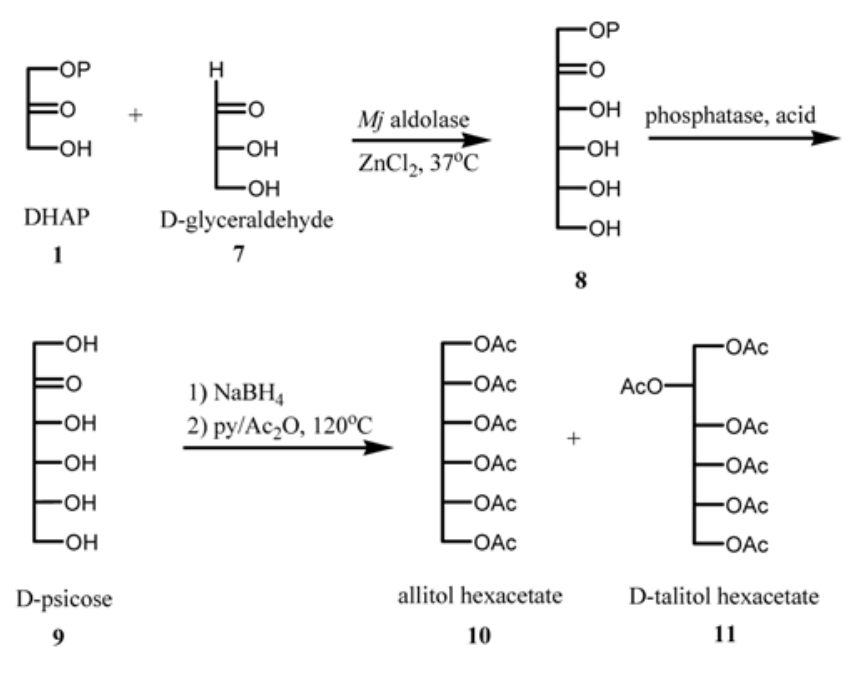

Scheme 2. $M j$ aldolase reaction with DHAP and Dglyceraldehyde.

reduction to prevent ketose to aldose isomerization. Since hydride $\left(\mathrm{H}^{-}\right)$can attack both sides of the carbonyl group in ketose, a mixture of two possible diastereomers is inevitably produced. Therefore, after dephosphorylation, reduction, and acetylation, the final products of each reaction should be adonitol (ribitol) pentacetate and D-arabitol (arabinitol) pentacetate from glycoaldehyde (Scheme 1), allitol hexacetate and Dtalitol hexacetate from D-glyceraldehyde (Scheme 2), and allitol hexacetate, D, L-talitol hexacetate, and dulcitol (galactitol) hexacetate from D, L-glyceraldehyde (Scheme 3).

To prepare standard samples, 3 pentoses (adonitol, D-arabitol, and xylitol) and 5-hexoses (allitol, D-talitol, D-sorbitol, Dmannitol, and dulcitol) were purchased from Sigma-Aldrich or TCI America and peracetylated with acetic anhydride in pyridine to yield peracetylated alditol derivatives (Fig. 1).

$M j$ aldolase catalysis was first examined using DHAP and glycoaldehyde, and the product obtained was dephosphorylated, reduced, and acetylated. GC analysis of the final product mixture and of standard alditol pentacetates are shown in Table 1. Final products were identified as mixtures of adonitol 

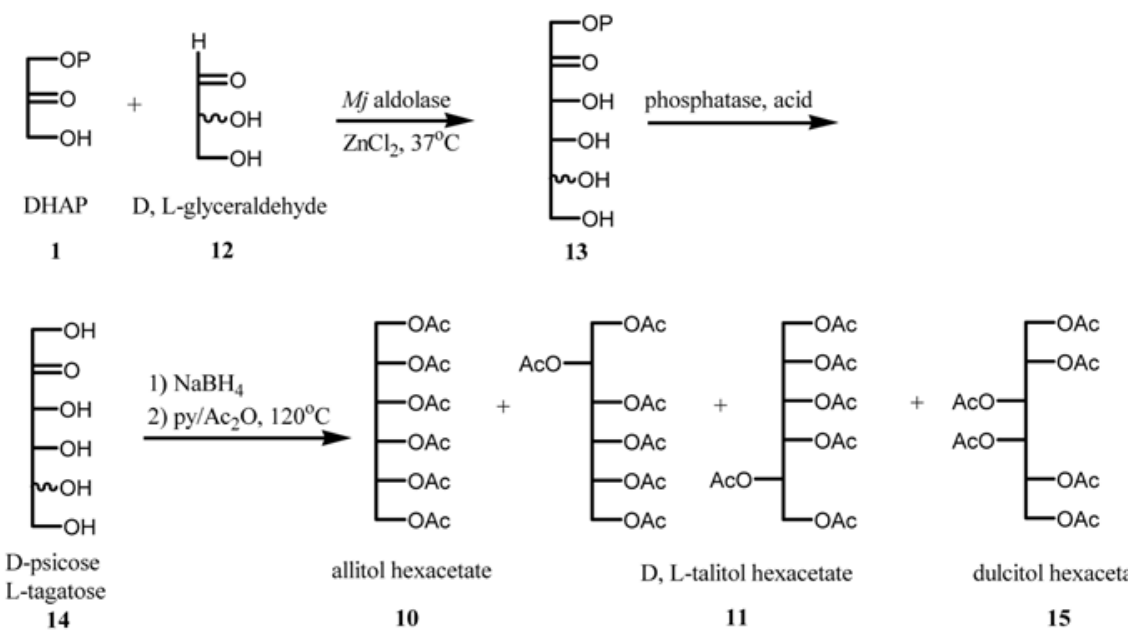

D-psicose

14

10

dulcitol hexacetate

15

Scheme 3. $M j$ aldolase reaction with DHAP and D, L-glyceraldehyde.

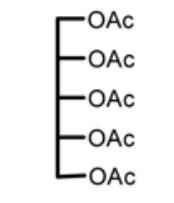

adonitol pentacetate

19

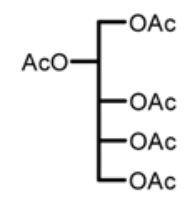

D-arabitol pentacetate

20

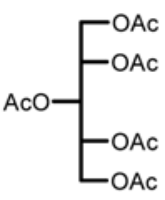

xylitol pentacetate

21
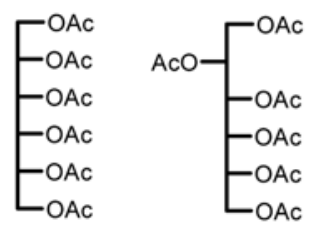

allitol hexacetate D-talitol hexaceto

27

28
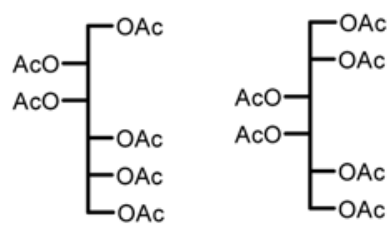

29

30

31

Fig. 1. Alditol derivatives for standard samples.

pentacetate and D-arabitol pentacetate by comparing the retention times of reaction products and standard samples. This result shows that the product of the aldol reaction between DHAP and glycoaldehyde was D-ribulose-1-phosphate, thus indicating that $M j$ aldolase is a fuculose-1-phosphate aldolase.

Using the same procedure, the products of reactions between DHAP and D-glyceraldehyde and between D, Lglyceraldehyde were analyzed by GC; results are shown in

Table 1. GC data of $M j$ aldolase reaction products from DHAP and glycoaldehyde

\begin{tabular}{lc}
\hline \multicolumn{1}{c}{ Sample } & Retention time \\
\hline Adonitol pentacetate & 17.715 \\
D-Arabitol pentacetate & 18.867 \\
Xylitol pentacetate & 24.867 \\
Reaction products of DHAP and glycoaldehyde & $17.793,18.987$ \\
\hline
\end{tabular}

Table 2. The table data indicated that the products were a mixture of D-allitol hexacetate and D-talitol hexacetate, and that the products of the reaction involving D, L-glyceraldehyde

Table 2. GC data of $M j$ aldolase reaction products starting from DHAP and glyceraldehydes

\begin{tabular}{lc}
\hline \multicolumn{1}{c}{ Sample } & $\begin{array}{c}\text { Retention } \\
\text { time }\end{array}$ \\
\hline Allitol hexacetate & 17.164 \\
D-Mannitol hexacetate & 19.152 \\
D-Talitol hexacetate & 19.463 \\
Dulcitol hexacetate & 20.977 \\
D-Sorbitol hexacetate & 23.520 \\
Reaction products of DHAP and D-glyceraldehyde & 17.249, \\
& 19.476 \\
Reaction products of DHAP and D, L-glyceralde- & 17.092, \\
hyde & 19.321, \\
& 21.020 \\
\hline
\end{tabular}


contained D-allitol hexacetate, D, L-talitol hexacetate, and dulcitol hexacetate. Again, these findings indicate that $M j$ aldolase converts D-glyceraldehyde to D-psicose-1-phosphate, and D, L-glyceraldehyde into a mixture of D-psicose-1phosphate and L-tagatose-1-phosphate, which also supports the notion that $M j$ aldolase is a fuculose-1-phosphate aldolase.

Summarizing, GC analysis was successfully applied to confirm that $M j$ aldolase is a fuculose-1-phosphate aldolase by identifying reaction products. Although GC is an dated technique, it is accurate and economic for the tentative identifications of carbohydrate derivatives. The present work will be extended to investigations of the substrate specificity and efficiency of $M j$ aldolase under different reaction conditions.

Acknowledgments This work was supported by a grant from the Soongsil University Fund, Seoul, Korea.

\section{References}

Calveras, J., Bujons, J., Parella, T., Crehuet, R., Espelt, L., Joglar, J. and Clapes, P. (2006) Influence of $\mathrm{N}$-amino protecting group on aldolase-catalyzed aldol additions of dihydroxyacetone phosphate to amino aldehydes. Tetrahedron 62, 2648-2656.

Choi, I-G., Cho, C. S., Cho, Y. and Yu, Y. G. (1998) Overproduction, purification, and characterization of heat-stable aldolase from Methanococcus jannaschii, a hyperthermophilic archaean. J. Biochem. Mol. Biol. 31, 130-134.

Dreyer, M. K. and Schulz, G. E. (1996) Catalytic mechanism of the metal-dependent fuculose aldolase from Escherichia coli as derived from the structure. J. Mol. Biol. 259, 458-466.

Espelt, L., Bujons, J., Parella, T., Calveras, J., Joglar, J., Delgado,
A. and Clapes, P. (2005) Aldol additions of dihydroxyacetone phosphate to $N$-Cbz-amino aldehydes catalyzed by L-fuculose1-phosphate aldolase in emulsion systems: inversion of stereoselectivity as a function of the acceptor aldehyde. Chem.A Euro. J. 11, 1392-1401.

Fessner, W-D., Gosse, C., Jaeschke, G. and Eyrisch, O. (2000) Enzymes in organic synthesis, Short enzymatic synthesis of Lfucose analogs. Euro. J. Org. Chem., 1, 125-132.

Franck C., Lahssen E. B., Thierry G., Laurence H., Jean B. and Marielle L. (2004) Improved Straightforward Chemical Synthesis of Dihydroxyacetone Phosphate through Enzymatic Desymmetrization of 2,2-Dimethoxypropane-1,3-diol. J. Org. Chem. 69, 9310-9312.

Jeyakanthan, J., Taka, J., Kikuchi, A. Kuroishi, C., Yutani, K., Shiro, Y. (2005) Purification, crystallization and preliminary $\mathrm{x}$ ray crystallographic study of the L-fuculose-1-phosphate aldolase (FucA) from Thermus thermophilus HB8. Acta Crystallogr., Sec. F: Struct. Biol. Crystal. Comm. 61, 10751077.

Kroemer, M., Merkel, I. and Schulz, G. E. (2003) Structure and Catalytic Mechanism of L-Rhamnulose-1-phosphate Aldolase. Biochemistry 42, 10560-10568.

Stephen W. B. F., Jennifer S. G., Alexandra B. M., Manoj C., Nathan J. W., Michael J. H., Carol A. F., Eric J. T. and James H. N. (2006) Mechanism of the Class I KDPG aldolase. Bioorg. Med. Chem. 14, 3002-3010.

Wong, C-H., Alajarin, R., Moris-Varas, F., Blanco, O., GarciaJunceda, E. (1995) Enzymic Synthesis of L-Fucose and Analogs. J. Org. Chem. 60, 7360-7363.

Yoon, H-S., Kwon, S-J., Han, M-S. Yu, Y-G. and Yoon, M-Y. (2001) Mutagenic characterization of a conserved functional amino acid in fuculose-1-phosphate aldolase from Methanococcus jannaschii, a hyperthermophilic archaea. J. Microbiol. Biotech. 11, 709-711. 